\title{
-Photogravure- \\ Epitope-grafted and Antigenized Antibodies Can Be Used for Versatile Vaccination Strategies to Induce Epitope-specific Immune Responses
}

\author{
Yoshihiro Kumagai \\ Department of Microbiology and Immunology, Nippon Medical School
}

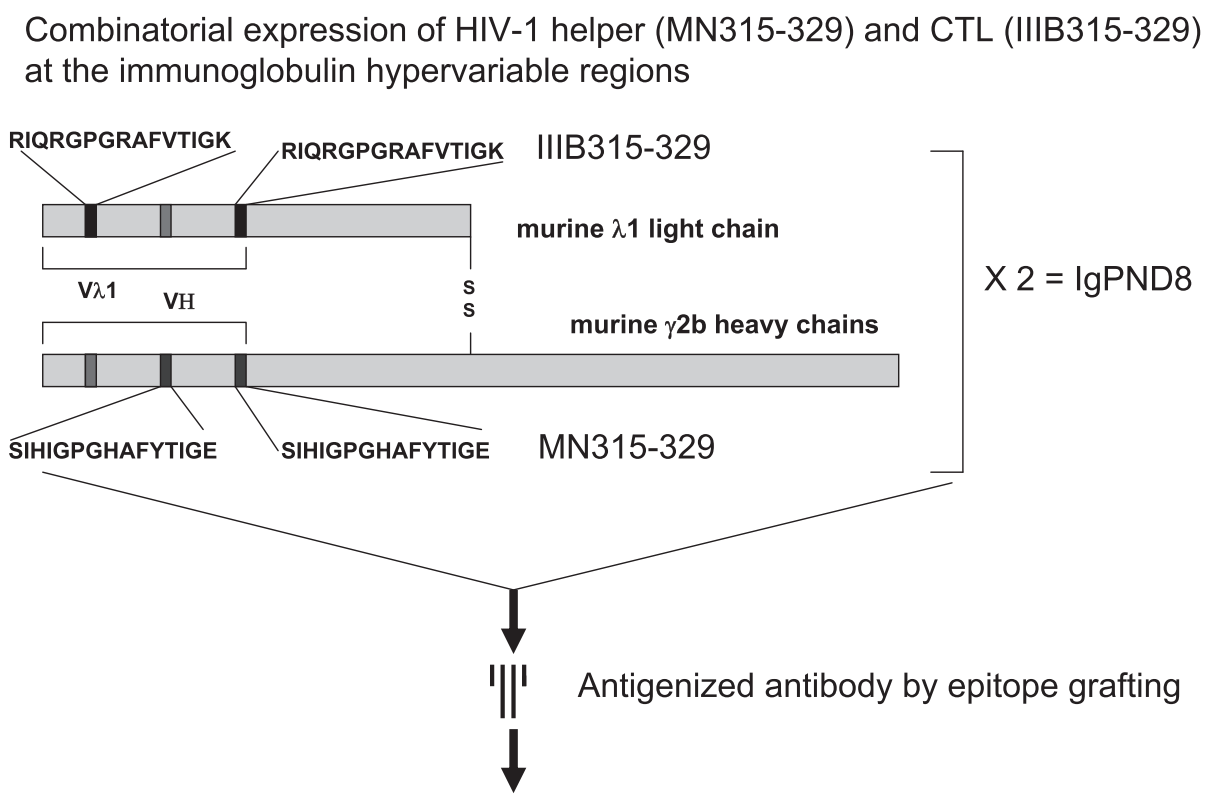

Bind to Fc receptors on antigen-presenting cells and processed to present the specific epitopes to T cells

Fig. 1

The epitopes (idiotopes) at the hypervariable complementarity-determining regions (CDRs) of immunoglobulin (Ig) molecules elicit idiotope-specific immune responses as idiotypic vaccines. The structural diversity of CDRs might mimic the variety of $\mathrm{B}$ and $\mathrm{T}$ cell epitopes of natural antigens. The V3 sequences of the human immunodeficiency virus (HIV) envelope protein (gp120) are the highly variable principal neutralizing determinants (PND) of HIV-1 infection in vitro and the sites to determine the cellular tropism of HIV-1. I have constructed HIV-1 V3 epitope libraries at the Ig hypervariable regions. The epitope-grafted Igs, which have V3sequences of human immunodeficiency virus envelope protein (gp120) at CDRs, could induce anti-V3 antibody responses and prime HIV-specific $\mathrm{T}$ cells in vivo. The analysis also provided evidence that the engineered Igs would be more efficiently presented to $\mathrm{T}$ cells by internalization with Fc receptors for Ig on antigen-presenting cells than synthetic V3 peptides. The processing of grafted V3 epitopes was quickly pursued from the plasma membrane to coated pits, multivesicular endosomes, and lysosomes, as shown in Figure 2.

Correspondence to Yoshihiro Kumagai, Department of Microbiology and Immunology, Nippon Medical School, 1-1-5 Sendagi, Bunkyo-ku, Tokyo 113-8602, Japan 
Time after addition of antigenized antibodies (IgPND8)

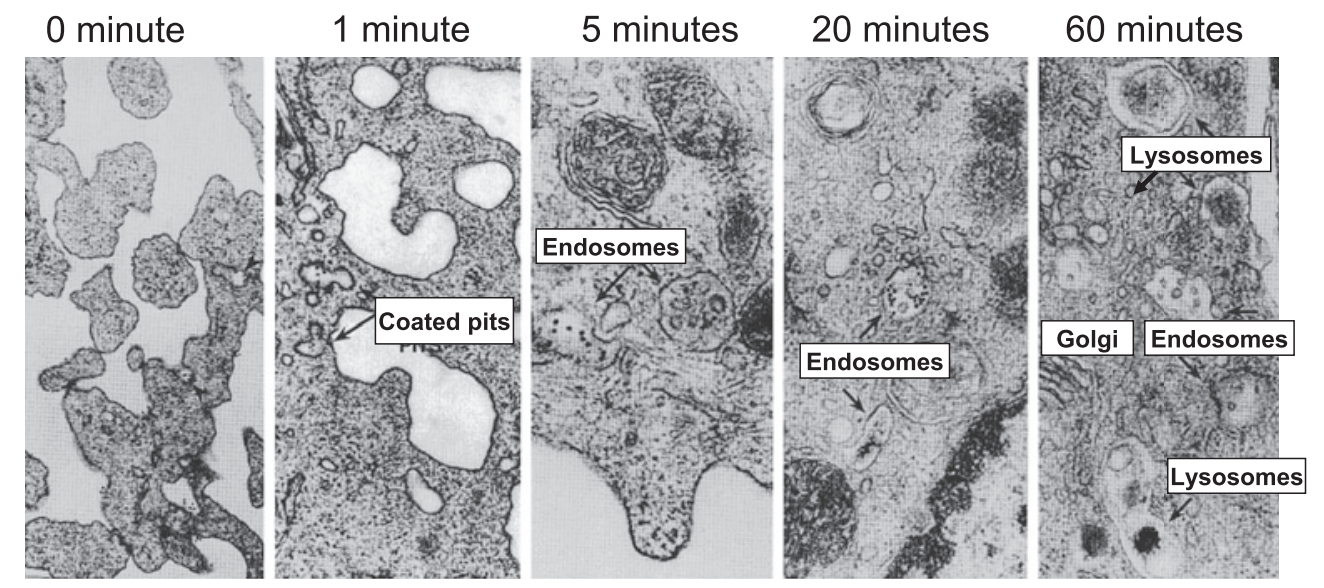

Localization of IgPND8 molecules (\%)

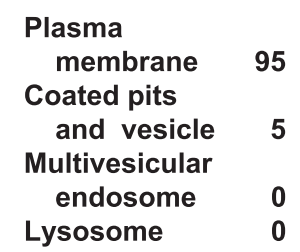

68

32

0
40

25

35

0

Fig. 2

Fig. 1 Molecular design of epitope-grafted antibodies

The sequence of SIHIGPGHAFYTIGE (residue 315-329 of HIV-1MNgp120) was used to replace the hypervariable region sequences of the murine $\gamma 2 \mathrm{~b}$ heavy chains, and the sequence RIQRGPGRAFVTIGK (residue 315-329 of HIV-1IIB gp120) was used for $\lambda 1$ light chains.

The mutated heavy chain variable region (VH) genes, from which the second CDR (CDR2) and the third CDR (CDR3) were deleted and replaced with the cognate peptide sequence in the correct frame, were ligated with XhoI linkers and subcloned at the XhoI sites in BIPE-neo vectors. The $\mathrm{V} \lambda 1$ gene was mutated in the same procedure at the first CDR (CDR1) and CDR3. The BIPE-neo vector was remodeled from the BMG-neo expression vector by replacing the metalothionine promoter region with the Ig heavy chain promoter (HindIII-NcoI) cloned from the rearranged genomic DNA of hybridoma cells producing template $\gamma 2 \mathrm{~b}$ heavy chains and the $\mu$ enhancer (XbaI$\mathrm{XbaI} 1.1 \mathrm{~kb}$ fragment). To express these genes, the non-Ig-secreting BALB/c plasmacytoma cell line SP2/0 was transfected with the vectors containing the mutated Ig $\gamma 2 \mathrm{~b}$ chain genes together with the mutated $\lambda 1$ chain constructs by electroporation. The culture supernatants of the transfectants were screened for the production of intact Ig context. The PND-grafted Igs were purified and tested for the ability to react with monoclonal antibodies (MoAb 50.1, Repligen Corp., Waltham, MA, USA) and polyclonal antibodies against the V3 peptides of gp120. The epitopegrafted Ig are designated as Ig-PND8 (8 copies of PND/intact Ig).

Fig. 2 Antigenized (HIV-1-V3 epitope-grafted) antibody molecules are internalized quickly at high efficiency in antigen presenting cells expressing immunoglobulin Fc receptors. The HIV-epitopegrafted Ig PND8 antibody proteins ( $10 \mu \mathrm{g} / \mathrm{mL}$ in RPMI1640 medium) were mixed with 1,000,000 of A20.J2 cells (antigen presenting cells), and the localization of grafted V3 epitopes was detected with colloidal gold-labeled anti-V3 mouse monoclonal antibodies in the plasma membrane, coated pit, multivesicular endosome, and lysosome fractions at 0,2, 5, 20, nd 60 minutes, respectively, after the addition of Ig PND8 molecules. The dense grains on electron microscopy show the localization of HIV epitopes carried by Ig PND8.

E-mail: ykminms@nms.ac.jp Journal Website (http://www.nms.ac.jp/jnms/) 